\title{
Percutaneous transluminal coronary angioplasty in patients with spasm superimposed on atherosclerotic narrowing
}

\author{
M E BERTRAND, J M LABLANCHE, J L FOURRIER, G TRAISNEL \\ From the Division of Cardiology, University Hospital of Lille, France
}

SUMMARY Of 552 patients undergoing percutaneous transluminal coronary angioplasty 102 had coronary artery spasm superimposed on atherosclerotic narrowing. Coronary angioplasty was successful in $97(95 \%)$. The patients were discharged on a regimen of nifedipine (40-60 mg/day). Seventy six patients were symptom free 6-8 months after the procedure. Restenosis was detected in $35 \%$ of patients. Coronary artery spasm was provoked in $38(44 \%)$ of the 87 patients who underwent an ergometrine maleate test. Twenty seven of the 34 patients with restenosis had a provocation test and coronary artery spasm was superimposed on restenosis in $22(81.5 \%)$.

Coronary angioplasty is feasible in patients with coronary artery spasm superimposed on atherosclerotic narrowing but the rate of restenosis is high and coronary artery spasm could have a role in the pathogenesis of restenosis.

It is now clearly established that coronary artery spasm has a major role in patients with Prinzmetal's variant angina. The coronary anatomy is variable in these patients, however, ranging from normal coronary vessels to severe three vessel disease. Coronary arterial spasm can be detected on angiographically normal coronary vessels or can be superimposed on fixed atherosclerotic narrowing: that is there is a functional component superimposed on the atherosclerotic plaque (organic component). In patients with severe coronary stenosis it seems logical to treat the two components. Coronary spasm can be prevented by calcium antagonists and elimination of the atherosclerotic plaque could improve coronary flow and relieve the symptoms. We have treated patients with coronary arterial spasm superimposed on significant atherosclerotic narrowing with percutaneous coronary angioplasty and calcium antagonists.

\section{Patients and methods}

PATIENTS

From December 1981 to September 1985, 522

Requests for reprints to Dr M E Bertrand, Service de Cardiologie B et Hémodynamique, Hopital Cardiologique, 59037 Lille Cedex, France.

Accepted for publication 27 April 1987 patients underwent percutaneous transluminal coronary angioplasty at the Lille Heart Institute. In this group 102 (95 men and 7 women; mean (SD) age 50 (9) years) had a dynamic coronary stenosis characterised by spontaneous (15) or provoked (87) coronary artery spasm superimposed on clinically significant coronary stenosis. Spasm was defined as a transient (reversible by intracoronary injection of isosorbide dinitrate) occlusion of the stenosis in which dilatation was to be attempted. Spasm proximal or distal to the stenosis site was not assessed.

Patients were assessed by a complete history, physical examination, and treadmill exercise test with Bruce's standard protocol. Angiographic examination included left ventricular cineangiography $\left(30^{\circ}\right.$ right anterior oblique projection) and selective right and left coronary arteriography performed from the femoral approach with Judkins-Bourassa's catheters; coronary arteries were filmed in five views for the left and four views for the right, including the craniocaudal sagittal angulated view.

If spontaneous spasm was not detected an ergometrine maleate provocation test was performed according to a protocol previously described. ${ }^{1}$ Coronary spasm was relieved by intracoronary injection of isosorbide dinitrate into the affected vessel. After intracoronary injection of nitrates all these patients had a high degree of residual stenosis. We carefully measured the lesions with automated digital callipers 
and a 6502 microprocessor. ${ }^{2}$ At least two measurements were taken in different projections and the results were expressed as a mean of these two values. All patients were premedicated with nifedipine (40 $\mathrm{mg}$ orally) and aspirin (500 $\mathrm{mg}$ ) on the day before coronary angioplasty. Coronary angioplasty was performed according to the technique initially described by Gruentzig et al. ${ }^{34}$ Until July 1983 we used Dilaca's catheter; after this we used a steerable guide wire balloon catheter. At the start of the procedure a bolus of $10000 \mathrm{IU}$ of heparin was administered intravenously. Some patients were given an intravenous infusion $(5 \mathrm{mg} / \mathrm{h})$ of isosorbide dinitrate. In others this drug was injected as required during the procedure. Primary success was defined as a $\geqslant 20 \%$ improvement in the luminal diameter without complications.

The patients were discharged on a regimen of dipyridamole $(150 \mathrm{mg})$ and 40 to $60 \mathrm{mg}$ of nifedipine daily. All the patients with a successful coronary angioplasty $(n=97)$ underwent a second coronary angiography 6-8 months later and an ergometrine maleate provocation test was performed in 87 patients.

\section{Results}

\section{CLINICAL FINDINGS BEFORE ANGIOPLASTY}

(Table 1).

Most patients experienced angina at rest. Forty six complained of episodes of pain at rest and 21 of them had typical Prinzmetal's variant angina defined as episodes of angina at rest associated with reversible ST segment elevation. Thirteen patients had epi-

Table 1 Clinical and angiographic data in 102 patients with spasm superimposed on atherosclerotic narrowing

\begin{tabular}{lc}
\hline Data & No \\
\hline No of patients & 102 \\
Age (yr)(SD) & $50(8)$ \\
Sex (M/F) & $95 / 7$ \\
Symptoms: & 21 \\
Prinzmetal's variant angina & 25 \\
Angina at rest & 20 \\
Effort angina & 13 \\
Mixed angina & 16 \\
Unstable angina & 7 \\
Atypical chest pain & \\
No of diseased vessels: & 80 \\
1 & 21 \\
2 & 1 \\
3 & 66 \\
Location of spasm: & 24 \\
LAD & 11 \\
RCA & 1 \\
LCX & \\
LAD + LCX &
\end{tabular}

LAD, left anterior descending artery; LCX, left circumflex coronary artery; RCA, right coronary artery. sodes of angina at rest and associated exertional angina. Twenty patients had typical angina on effort. Sixteen patients were admitted to the coronary care unit with unstable angina. Seven patients complained of atypical chest pain.

Coronary angiography showed the high frequency of single vessel disease $(82 / 102=80 \%)$. Twenty patients had double or triple vessel disease. The mean (SD) reduction of luminal diameter was 72 $(13 \%)$.

Spontaneous spasm was found in 15 patients and spasm was provoked in 87 patients. Spasm occurred in the left anterior descending coronary artery in 66 patients, in the right coronary artery in 24 , and in the circumflex branch in 11. In one patient there was simultaneous spasm in the left anterior descending coronary artery and the circumflex artery.

\section{CORONARY ANGIOPLASTY PROCEDURE}

Coronary angioplasty was attempted in 111 vessels. Table 2 lists the vessels attempted and the details of the procedure. In four patients it was impossible to cross the lesion. One of these patients needed an emergency bypass operation, which was performed without complication. The narrowing was dilated in 98 patients but one had a poor result with $75 \%$ residual stenosis and myocardial infarction occurred within 48 hours of the procedure. At the end of the procedure in a few patients we noted transient narrowing of the distal part of the vessel, beyond the dilated stenosis. This was easily reversed with glyceryl trinitrate. This spasm was provoked by mechanical stimulation of the vessel by the steerable guide wire. In patients with successful coronary angioplasty the narrowing was significantly reduced from $72(13 \%)$ to $21(48 \%)$ and transtenotic pressure gradient was reduced from $57(23)$ to $8(8) \mathrm{mm} \mathrm{Hg}$.

Coronary angioplasty was successful in 97 $(95 \cdot 1 \%)$ of the 102 patients. The complication rate

Table 2 Percutaneous transluminal coronary angioplasty procedure in 97 patients

\begin{tabular}{ll}
\hline & No \\
\hline Dilated vessel: & \\
LAD & 76 \\
RCA & 24 \\
LCX & 11 \\
Stenosis (\%): & $72(13)$ \\
Before PTCA & $21(48)$ \\
After PTCA & $57(23)$ \\
Transtenotic gradient (mm Hg): & $8(8)$ \\
Before PTCA & After PTCA \\
\hline LAD, left anterior descending artery; LCX, left circumflex \\
coronary artery; PTCA, percutaneous transluminal coronary angio- \\
plasty; RCA, right coronary artery.
\end{tabular}


was low: it included one $(0.9 \%)$ myocardial infarction and one emergency bypass operation without sequelae.

\section{CLINICAL AND ANGIOGRAPHIC FOLLOW UP (Table 3)}

Six to eight months after coronary angioplasty we re-examined the 97 patients clinically and angiographically. Seventy six patients were symptom free. Four had angina pain at rest and seven complained of pain during effort and at rest; only five of them had effort angina alone and five had unstable angina. Restenosis was defined as a loss of $50 \%$ of the initial gain achieved at percutaneous transluminal coronary angioplasty. Thirty four (35\%) had restenosis. Only $15 \%$ of patients with angiographic evidence of restenosis were symptom free. The provocation test was repeated during coronary angiography in 87 . patients and coronary artery spasm was induced in $38(44 \%)$. Twenty seven of the 34 patients with restenosis had a provocation test. The test was not performed in seven patients because four of them had occlusion of the dilated segment and three had severe triple vessel disease. Twenty two (81.5\%) patients had spasm at the site of the dilated coronary segment that was superimposed on restenosis.

\section{Discussion}

$\therefore$ This study is the fourth report ${ }^{5-7}$ of coronary angioplasty-in a large series of patients with coronary artery :spasm superimposed on atherosclerotic nar.rowing. In 1982, David et al demonstrated that coronary angioplasty. was technically feasible in patients with Prinzmetal's variant angina and that the fixed obstruction can be treated by this technique. ${ }^{5}$ Hollman $e t$ al reviewed the clinical course of five patients with spasm superimposed on the coronary segment treated by angioplasty. ${ }^{6}$ Corcos et al described a series of 21 patients with variant angina treated with coronary angioplasty. ${ }^{7}$ All these studies clearly demonstrated that the results of coronary angioplasty in these patients are less satisfactory than in other groups.

Table 3. Data at follow up in 97 patients

\begin{tabular}{ll}
\hline & No \\
\hline Restenosis & 34 \\
Symptom free & 76 \\
Symptoms: & 4 \\
Angina at rest & 7 \\
Mixed angina & 5 \\
Effort angina & 5 \\
Unstable angina & 87 \\
Provocation test & 38 \\
Coronary spasm & $22 / 27$ \\
Restenosis + spasm & \\
\hline
\end{tabular}

Five of the 13 patients in David et al's study had restenosis and two others developed severe lesions adjacent to the site of dilatation. ${ }^{5}$ Four of the five patients in Hollman et:al's study did not respond to treatment with calcium antagonists and subsequently developed restenosis. ${ }^{6}$ The restenosis rate was $47 \%$ in the series of Corcos et al.? Coronary artery spasm persisted in $44 \%$ of our patients despite treatment with calcium channel blockers; but it should be noted that these drugs were discontinued 12 to 18 hours before follow up angiography.

The persistence of spasm is not totally unexpected. Experimental studies have shown that after angioplasty the endothelium is denuded and damaged. $^{8}$ : This could lead to the release of platelet factors such as adenosine diphosphate, platelet growth factor 4, and thromboxane, which is a potent vasoconstrictor. Therefore, the vasomotor reactivity of the dilated segment could be increased. This hypothesis is supported by the study of a group of 202 patients who had a negative ergometrine provocation test before percutaneous transluminal coronary angioplasty. Forty two $(20.7 \%)$ of them had a coronary arterial spasm induced by ergometrine at angiographic follow up. It could be that the spastic disease was quiescent at the initial evaluation, but it is also possible that angioplasty denudes the vessel of endothelium and makes it more reactive. The restenosis rate in the present study was $35 \%$; this is higher than the rate in other groups of patients treated with coronary angioplasty. Moreover, $81.5 \%$ of patients with restenosis had spasm superimposed on the narrowing.

The finding that the restenosis rate is higher in patients with variant angina than in patients with a fixed coronary obstruction supports the hypothesis that persistence of coronary spasm has a role in the pathogenesis of restenosis in patients with variant angina. Restenosis is a common complication of coronary angioplasty but the mechanisms of this complication are still unclear. Chahine and Raizner in 1977 and Marzilli et al (in 1980) suggested that recurrent vasospastic episodes in an angiographically normal or minimally diseased coronary segment could result in a more rapid progression of the atherosclerotic process. ${ }^{910}$

.. In our study patients were treated with $\mathbf{4 0}$ to $\mathbf{6 0}$ mg of nifedipine daily. Corcos et al demonstrated restenosis only in patients not treated with calcium antagonists. A randomised study by the same group however; did not find a significant difference in the restenosis rate in patients treated with calcium antagonists and those who were not. ${ }^{11}$ More recently, Whitworth et al in a double blind randomised study of 241 patients did not find that nifedipine significantly reduced the restenosis rate after suc- 
cessful coronary angioplasty. ${ }^{12}$ Further studies are needed to establish adequate medical treatment to prevent restenosis.

\section{References}

1 Bertrand ME, Lablanche JM, Tilmant PY, et al. Frequency of provoked coronary arterial spasm in 1089 consecutive patients undergoing coronary arteriography. Circulation 1982;65:1299-306.

2 Bertrand ME, Lablanche JM, Thieuleux FA, Fourrier $\mathrm{JL}$, Traisnel G. Mesure du diamètre des artères et des sténoses coronaires. Présentation de deux méthodes. Ann Cardiol Angeiol (Paris) 1985;34:615-9.

3 Gruentzig A, Senning A, Siegenthaler WE. Non operative dilation of coronary artery stenosis. Percutaneous transluminal coronary angioplasty. $N$ Engl J Med 1979;301:61-3.

4 Gruentzig AR. Technique of percutaneous transluminal coronary angioplasty. In: Hurst JW, ed. The heart. New York: McGraw Hill 1982:1904-10.

5 David PR, Waters DD, Scholl JM, et al. Percutaneous transluminal coronary angioplasty in patients with variant angina. Circulation 1982;66:695-702.

6 Hollman J, Austin GE, Grunentzig AR, Douglas JS Jr,
King SB III. Coronry spasm at the site of angioplasty in the first two months after successful percutaneous coronary angioplasty. J Am Coll Cardiol 1983;2: 1039-45.

7 Corcos T, David PR, Bourassa MG, Guiteras P, Robert J, Mata LA. Percutaneous transluminal coronary angioplasty for the treatment of variant angina. J Am Coll Cardiol 1985;5:1046-54.

8 Faxon DP, Sanborn TA, Handenschild CC, et al. Effect of antiplatelet therapy on restenosis after experimental angioplasty. Am J Cardiol 1984;53:72C-6C.

9 Chahine RA, Raizner AE. Another look at Prinzmetal's variant angina. Eur J Cardiol 1977;6:71-4.

10 Marzilli M, Goldstein S, Trivella MG, et al. Some clinical considerations regarding the relation of coronary vasospasm to coronary atherosclerosis. A hypothetical pathogenesis. Am J Cardiol 1980;45:882-6.

11 Corcos T, David PR, Val PG, et al. Failure of diltiazem to prevent restenosis after percutaneous transluminal coronary angioplasty (PTCA). Am Heart J 1985;109: 925-31.

12 Whitworth HB, Roubin GS, Hollman J, et al. Effects of nifedipine on recurrent stenosis after percutaneous transluminal coronary angioplasty. J Am Coll Cardiol 1986;8:1271-6. 